This item was submitted to Loughborough's Research Repository by the author.

Items in Figshare are protected by copyright, with all rights reserved, unless otherwise indicated.

\title{
Delamination propagation under high loading rate
}

PLEASE CITE THE PUBLISHED VERSION

https://doi.org/10.1016/j.compstruct.2020.112734

PUBLISHER

Elsevier BV

VERSION

AM (Accepted Manuscript)

PUBLISHER STATEMENT

This paper was accepted for publication in the journal Composite Structures and the definitive published version is available at https://doi.org/10.1016/j.compstruct.2020.112734

\section{LICENCE}

CC BY-NC-ND 4.0

\section{REPOSITORY RECORD}

Chen, Tianyu, Christopher Harvey, Simon Wang, and Vadim Silberschmidt. 2020. "Delamination Propagation Under High Loading Rate”. Loughborough University. https://hdl.handle.net/2134/12789596.v1. 


\title{
Delamination propagation under high loading rate
}

\author{
Tianyu Chen ${ }^{\mathrm{a}}$, Christopher M. Harvey ${ }^{\mathrm{a}, \mathrm{b}, *}$, Simon Wang, ${ }^{\mathrm{a}, \mathrm{b}}$, Vadim V. Silberschmidt ${ }^{\mathrm{c}}$ \\ ${ }^{a}$ Department of Aeronautical and Automotive Engineering, Loughborough University, \\ Loughborough, Leicestershire LE11 3TU, UK \\ ${ }^{b}$ School of Mechanical and Equipment Engineering, Hebei University of Engineering, Handan 056038, China \\ ${ }^{c}$ Wolfson School of Mechanical, Electrical and Manufacturing Engineering, Loughborough University, \\ Loughborough, Leicestershire LE11 3TU, UK
}

\begin{abstract}
Analytical theory for the dynamic delamination behavior of a double cantilever beam (DCB) under high loading rate is developed. Structural vibration and wave dispersion are considered in the context of Euler-Bernoulli beam theory. The theory is developed for both initiation and propagation of delamination in mode I. Two solutions for the energy release rate (ERR) are given for a stationary delamination: an accurate one and a simplified one. The former is based on global energy balance, structural vibration and wave dispersion; the latter is 'local' since it is based on the crack-tip bending moment. For the simplified solution to be accurate, sufficient time is needed to allow the establishment of all the standing waves. For a propagating delamination, a solution for the ERR is derived using the same simplification with the cracktip bending moment. The obtained ERR solutions are verified against experimental data and results from finite-element simulations, showing excellent agreement. One valuable application of the developed theory is to determine a material's dynamic loading-ratedependent delamination toughness by providing the analytical theory to post-process test results of dynamic DCB delamination.
\end{abstract}

Keywords: Double cantilever beam dynamics; Delamination propagation; Dynamic energy release rate; Dynamic delamination toughness; Vibration

\footnotetext{
* Corresponding Author
}

Email addresses: t. chen3@lboro.ac.uk (Tianyu Chen), c.m.harvey@lboro.ac.uk (Christopher M. Harvey), s.wang@lboro.ac.uk (Simon Wang), 


\begin{tabular}{|c|c|}
\hline Nomenclature & \\
\hline$a$ & Delamination length \\
\hline$\dot{a}$ & Delamination propagation speed \\
\hline$A$ & Area of cross-section of beam \\
\hline$A_{0}$ & Delamination area \\
\hline$b$ & Width of beam \\
\hline$C_{\mathrm{g}}^{i}$ & Group speed of the $i$ th mode flexural wave \\
\hline$C_{\mathrm{p}}^{1}$ & Phase speed of the first mode flexural wave \\
\hline$C_{\mathrm{p}}^{i}$ & Phase speed of the $i$ th mode flexural wave \\
\hline$E$ & Young's modulus \\
\hline$E_{\mathrm{vib}}$ & Total energy density due to vibration \\
\hline$E_{\mathrm{vib}}^{i}$ & $i$ th mode energy density \\
\hline$f_{i}$ & Reduction factor for $i$ th vibration mode's energy flux due to dispersion \\
\hline$F(\Omega)$ & Energy flux \\
\hline$F_{\text {vib }}(\Omega)$ & Energy flux due to vibration \\
\hline$F_{\mathrm{vib}}^{i}(\Omega)$ & $i$ th mode wave energy flux \\
\hline$G$ & Energy release rate \\
\hline$G_{\text {stationary }}^{\text {global }}$ & Energy release rate for stationary delamination using global energy balance \\
\hline$G_{\text {stationary }}^{\text {local }}$ & Energy release rate for stationary delamination using local method \\
\hline$G_{\mathrm{st}}^{\mathrm{K}}$ & ERR component due to kinetic energy of quasi-static motion \\
\hline$G_{\mathrm{st}}^{\mathrm{U}}$ & ERR component due to strain energy of quasi-static motion \\
\hline$G_{\mathrm{vib}}$ & ERR component due to vibration \\
\hline$G_{\mathrm{vib}}^{i}$ & ERR component due to $i$ th vibration mode \\
\hline$h$ & Thickness of beam \\
\hline$I$ & Second moment of area of beam \\
\hline$K$ & Kinetic energy \\
\hline$n$ & Relative time \\
\hline$t$ & Time \\
\hline$U$ & Strain energy \\
\hline$v$ & Applied constant opening velocity \\
\hline$w(x, t)$ & Total transverse deflection \\
\hline$W_{\text {ext }}$ & Work done by external force \\
\hline$\rho$ & Density \\
\hline$\omega$ & Angular frequency \\
\hline Abbreviations & \\
\hline DAT & Delamination arrest toughness \\
\hline DCB & Double cantilever beam \\
\hline DIT & Delamination initiation toughness \\
\hline ERR & Energy release rate \\
\hline FEM & Finite-element method \\
\hline ITLS & Interfacial thick level set \\
\hline VCCT & Virtual crack closure technique \\
\hline
\end{tabular}




\section{Introduction}

Double cantilever beams (DCBs) are widely used in experiments to determine the delamination fracture toughness of laminates under pure-mode-I loading. For quasi-static loading, there is a standard test method to determine the mode-I delamination fracture toughness [1]. For high loading rate, however, there are to the authors' knowledge, relatively few studies and no standard test method. This is the current situation despite the fact that dynamic delamination fracture toughness, a critical material property in various applications of layered materials and joints undergoing dynamic loads, is generally loading-rate-dependent $[2][3][4]$. Some examples, among many others, include drilling or cutting of laminated composites; damage in laminated composite structures or joints exposed to vibration and fatigue in aerospace, automotive, naval and energy applications; unstable spallation of protective coatings under in-plane residual stresses; impact of layered material systems such as vehicle and body armor.

Refs. [5][6][7] are three key early studies on dynamic delamination. They all used a global energy balance approach, but without considering vibration, and so provide effectively 'smoothed' solutions for energy release rate (ERR). The vibration experienced under transient or varying external loads can, however, often be significant [6][7][8][9][10][11], and in these cases ignoring vibration is not an acceptable approximation. The vibration of DCBs results in additional transverse deflection around the crack tip, which can sometimes cause the delamination to close, giving a reduced ERR, and can sometimes cause the delamination to open, giving an increased ERR. These phenomena are responsible for an oscillating ERR as demonstrated by experiment in Ref. [8] and by numerical studies in Ref. [9].

Accurately determining dynamic ERR under high loading rate is essential for proper understanding of dynamic delamination behavior, as well as for prediction of the dynamic delamination toughness of a given material and the extent of delamination. To the authors' knowledge, this has not yet been achieved analytically with the consideration of vibration; rather, only computationally intensive numerical simulation were employed [9][12][13]. Additionally, such an analytical theory would be helpful in understanding the 'stick-slip' delamination growth behavior [6][7][10] and in explaining whether a high loading rate increases or decreases the delamination toughness of materials, which is debated in Ref. [11].

The authors have previously developed an analytical theory for stationary delamination on both rigid and non-rigid interfaces [14][15][16], and the developed analytical solutions were based on a non-moving boundary condition to derive the dynamic ERR with account for 
vibration and wave propagation. Those analytical solutions can be readily used to measure the delamination initiation toughness for their respective cases, but they cannot be used to study delamination propagation problems under dynamic loads. In this work, the analytical theory for delamination propagation is developed with account for vibration and wave propagation. To the authors' knowledge, this has not ever been achieved analytically before; rather, only by computationally intensive numerical simulation. In addition, in order to solve the problem, a moving boundary condition problem is solved in a novel way using engineering approximations. The developed theory can be used to study delamination propagation, arrest and re-initiation, and can be applied to measure delamination initiation toughness (DIT) and delamination arrest toughness (DAT). Another valuable application afforded by this development for delamination propagation is assessment of a material's dynamic loading-ratedependent delamination toughness from test results of dynamic DCB delamination.

The theory is developed in Section 2, where in order to derive the analytical theory of delamination propagation, the author's existing theory for stationary delamination [14][15][16] is first simplified, so that it can be more readily applied to delamination propagation, and rigorously verified. The simplification technique is then used to develop the analytical theory of delamination propagation under high loading rate. The theory is verified against experimental data and finite-element method (FEM) simulations in Section 3. Conclusions are given in Section 4.

\section{Theory}

\subsection{Stationary delamination}

Consider the DCB geometry shown in Fig. 1a. The delamination length is $a$ and the thickness of each DCB arm is $h$. Each arm is subjected to a constant opening velocity $v$ from $t=0$, so $v t$ is the opening displacement applied to each DCB arm. Both arms are thin, with $h \square a$, and so classical Euler-Bernoulli beam theory applies. Small displacements without developed longitudinal forces are assumed. Under these assumptions, one DCB arm can be considered in analysis, with the effective boundary conditions shown in Fig. 1b. This boundary condition is in accordance with conventional methods used to determine the ERR of a symmetric DCB [17]. The origin of the $x$ coordinate for the single-arm analysis is at the free end and positive towards the right (Fig. 1b), which is the direction of delamination propagation; the transverse deflection is in the $x-z$ plane. 
a)

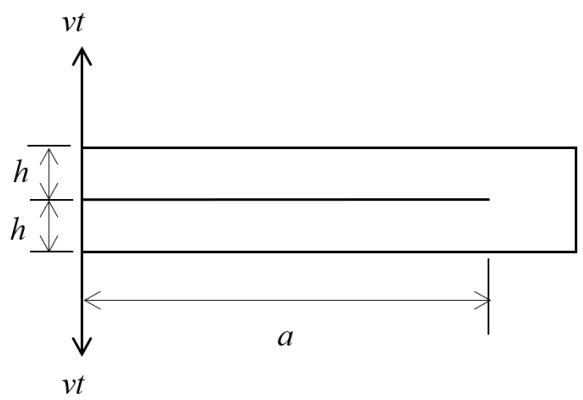

b)

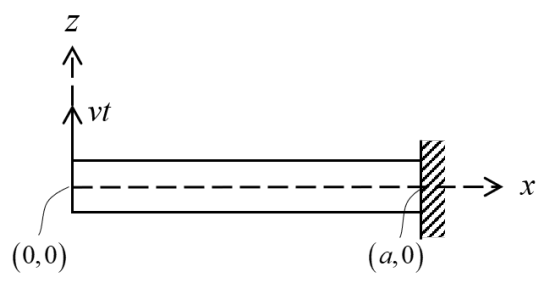

Fig. 1. (a) Symmetric double cantilever beam; (b) effective boundary condition of single-arm analysis

By coordinate transformation of the solution in Ref. [15], under plane-stress conditions the deflection of the half DCB with the effective boundary condition shown in Fig. $1 \mathrm{~b}$ is

$$
w(x, t)=v a^{2} \sqrt{\frac{\rho A}{E I}} \sum_{i=1}^{\infty} \frac{\Lambda_{i}}{\lambda_{i}^{3}} \phi_{i}(a-x) \sin \left(\omega_{i} t\right)+\left(\frac{x^{3}}{2 a^{3}}-\frac{3 x}{2 a}+1\right) v t,
$$

where

$$
\phi_{i}(x)
$$

is

the

mode

shape,

$\phi_{i}(x)=\cosh \left(\lambda_{i} x / a\right)-\cos \left(\lambda_{i} x / a\right)-\sigma_{i}\left[\sinh \left(\lambda_{i} x / a\right)-\sin \left(\lambda_{i} x / a\right)\right], \lambda_{i}$ is determined by the frequency equation $\tanh \left(\lambda_{i}\right)-\tan \left(\lambda_{i}\right)=0 \quad$ giving $\quad \omega_{i}=\lambda_{i}^{2} / a^{2} \sqrt{E I /(\rho A)} \quad, \quad$ and $\Lambda_{i}=(-1)^{i} \sqrt{\sigma_{i}^{2}+1}+\sqrt{\sigma_{i}^{2}-1}$ with $\sigma_{i}=\left[\cosh \left(\lambda_{i}\right)-\cos \left(\lambda_{i}\right)\right] /\left[\sinh \left(\lambda_{i}\right)-\sin \left(\lambda_{i}\right)\right]$. Note that for plane-strain beams, $E$ must be replaced by $E /\left(1-v^{2}\right)$ here and throughout this article.

The deflection in Eq. (1) has two terms: the first term is the local free vibration $w_{\mathrm{fv}}(x, t)$, which is due to the initial applied opening velocity; the second term is the quasi-static motion component, which is the distribution of the applied free-end displacement $v t$ along the beam length through the shifting function. For the reader's convenience, the original derivation from Ref. [15] is contained in brief in Appendix A. Note that the physical interpretation of the parameter $\Lambda_{i}$ is the coupling of local vibration and quasi-static motion, which is used to explain the contribution of each vibration mode to the dynamic ERR in Section 2.1.1.

\subsubsection{Dynamic ERR for stationary delamination}

The strain energy of the half DCB in Fig. 1b is [15]

$$
U=\frac{3}{2} \frac{E I v^{2} t^{2}}{a^{3}}+\frac{1}{2} \rho A a v^{2} \sum_{i=1}^{\infty} \frac{\Lambda_{i}^{2}}{\lambda_{i}^{2}} \sin ^{2}\left(\omega_{i} t\right)
$$


and its kinetic energy is

$$
K=\frac{1}{2} \rho A a v^{2} \sum_{i=1}^{\infty} \frac{\Lambda_{i}^{2}}{\lambda_{i}^{2}} \cos ^{2}\left(\omega_{i} t\right)-\rho A a v^{2} \sum_{i=1}^{\infty} \frac{\Lambda_{i}^{2}}{\lambda_{i}^{2}} \cos \left(\omega_{i} t\right)+\frac{33}{280} \rho A a v^{2} .
$$

The total mechanical energy of the half DCB at a given time $t$ is therefore

$$
\begin{aligned}
\Pi & =U+K \\
& =\frac{3}{2} \frac{E I v^{2} t^{2}}{a^{3}}+\frac{33}{280} \rho A a v^{2}+\frac{1}{2} \rho A a v^{2} \sum_{i=1}^{\infty} \frac{\Lambda_{i}^{2}}{\lambda_{i}^{2}}-\rho A a v^{2} \sum_{i=1}^{\infty} \frac{\Lambda_{i}^{2}}{\lambda_{i}^{2}} \cos \left(\omega_{i} t\right) .
\end{aligned}
$$

Note that the first and second terms in Eq. (4) are the strain and kinetic energies, respectively, due to quasi-static motion; and last two terms are the energy due to vibration. To aid in the following development, these contributions are denoted by $\Pi_{\mathrm{st}}^{\mathrm{U}}, \Pi_{\mathrm{st}}^{\mathrm{K}}$ and $\Pi_{\mathrm{vib}}$ respectively.

The dynamic ERR of the DCB shown in Fig. 1a (i.e. comprising two of the single arms in Fig. 1b with equal and opposite displacements) can be expressed as the sum of corresponding contributions, that is, $G=G_{\mathrm{st}}^{\mathrm{U}}+G_{\mathrm{st}}^{\mathrm{K}}+G_{\mathrm{vib}}$, where $G_{\mathrm{st}}^{\mathrm{U}}$ and $G_{\mathrm{st}}^{\mathrm{K}}$ are the ERR components due to the strain and kinetic energies of quasi-static motion; and $G_{\text {vib }}$ is ERR component due to vibration. Note that $G_{\text {st }}^{\mathrm{U}}$, which does not include any dynamic effect, is the conventional ERR for quasi-static loading. Also note that the ERR due to quasi-static motion, $G_{\mathrm{st}}=G_{\mathrm{st}}^{\mathrm{U}}+G_{\mathrm{st}}^{\mathrm{K}}$, is the dynamic ERR in Refs. [5][6][7], which provides a 'smoothed' ERR without the ERR component due to vibration. The ERR due to quasi-static motion is calculated as

$$
G_{\mathrm{st}}=-\frac{d}{d A_{0}}\left(\Pi_{\mathrm{st}}^{\mathrm{U}}+\Pi_{\mathrm{st}}^{\mathrm{K}}\right)=\frac{9 E I v^{2} t^{2}}{b a^{4}}-\frac{33 \rho A v^{2}}{140 b} .
$$

The ERR component due to vibration $G_{\text {vib }}$, however, cannot be determined as $-d \Pi_{\text {vib }} / d A_{0}$ . Doing so results in $G_{\text {vib }}$ being divergent with increasing vibration mode numbers, as discovered and solved in Refs. [15][16]. The reason for the divergence is that $-d \Pi_{\text {vib }} / d A_{0}$ overestimates the energy supplied to the crack tip at a given time by overlooking that beams as 1D waveguides are highly dispersive, that is, flexural waves with different frequencies travel with different wave speeds. Calculating $G_{\text {vib }}$ as $-d \Pi_{\text {vib }} / d A_{0}$ ignores this dispersive property and assumes that all the waves arrive at crack tip simultaneously. 
Freund [18] proposed that the dynamic ERR can be calculated as $G=F(\Omega) / \dot{A}_{0}$, where $F(\Omega)$ is the energy flux into a contour around the crack tip. Accordingly, the ERR component due to vibration $G_{\text {vib }}$ is calculated as

$$
G_{\text {vib }}=\frac{F_{\text {vib }}(\Omega)}{\dot{A}_{0}},
$$

where $F_{\text {vib }}(\Omega)$ is the energy flux due to vibration through the contour $\Omega$ shown in Fig. 2, in which $\varepsilon \square a$.

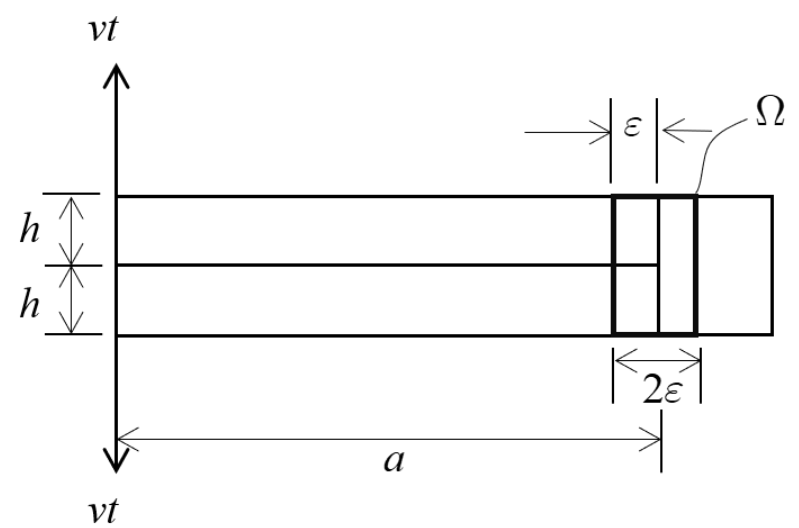

Fig. 2. Crack tip contour $\Omega$

The energy flux due to vibration through the contour $\Omega$ can be calculated as $F_{\text {vib }}(\Omega)=E_{\text {vib }} C_{\mathrm{p}}^{1}$, where $E_{\text {vib }}$ is the total energy density due to vibration and $C_{\mathrm{p}}^{1}$ is the phase speed of the first mode flexural wave. This is because the first mode flexural wave modulates all the other waves with higher frequencies [19], and, thus, the average speed of total energy flux is $C_{\mathrm{p}}^{1}$. To determine the total energy density due to vibration $E_{\text {vib }}$, a small region $a-\varepsilon<x<a$ in front of the crack tip is considered (the energy density in the small region $a<x<a+\varepsilon$ behind the crack tip is zero due to the effective boundary condition). The sign of the spatial distribution of free vibration in this region is proportional to $\Lambda_{i}$, which alternates with vibration mode numbers since $\Lambda_{i} \approx(-1)^{i} \sqrt{2}$ (see Appendix A). Note that for each flexural wave, its free-vibration component has contributions from both the space and time domains. The contribution from the space domain is the spatial distribution of free vibration, which is proportional to $\Lambda_{i}$; and the contribution from the time domain oscillates with $\sin \left(\omega_{i} t\right)$. Therefore, $\Lambda_{i} \approx(-1)^{i} \sqrt{2}$ means that the contribution from the space domain of 
flexural waves with odd mode numbers tend to close the delamination and decrease the total energy density in the space domain, while those with even mode numbers tend to open the delamination and increase the total energy density. Further details can be found in Refs. [15][16]. The total energy density due to vibration $E_{\text {vib }}$ in the contour is therefore

$$
E_{\mathrm{vib}}=\sum_{i=1}^{\infty}(-1)^{i} E_{\mathrm{vib}}^{i}
$$

where $E_{\mathrm{vib}}^{i}$ is the $i$ th mode energy density.

Further examination reveals that $\Lambda_{i}$ comes from the initial modal velocity (see Appendix A) [15], which is the coupling of the normal mode (representing the free vibration) and the quasi-static motion along the beam (via the shifting function). The physical interpretation of Eq. (7) is therefore that when the spatial velocity of free vibration of one flexural wave, represented by the normal mode, is in the same direction as the applied opening velocity, this flexural wave opens the delamination and increases the energy density in the contour in the space domain. Likewise, when the spatial velocity of free vibration of one flexural wave, represented by the normal mode, is in the opposite direction to the applied opening velocity, this flexural wave closes the delamination and decreases the energy density in the contour in the space domain.

The energy flux of the $i$ th mode wave $F_{\text {vib }}^{i}(\Omega)$ is $F_{\text {vib }}^{i}(\Omega)=E_{\mathrm{vib}}^{i} C_{\mathrm{g}}^{i}$, where $C_{\mathrm{g}}^{i}$ is the wave's group speed (since the energy of a wave propagates at its group speed [20]). Since $F_{\text {vib }}(\Omega)=E_{\text {vib }} C_{\mathrm{p}}^{1} \quad$ (see above) and using Eq. (7), this therefore gives $F_{\text {vib }}(\Omega)=\sum_{i=1}^{\infty}(-1)^{i} F_{\text {vib }}^{i}(\Omega) f_{i}$ where $f_{i}=C_{\mathrm{p}}^{1} / C_{\mathrm{g}}^{i}$. The $i$ th mode wave energy flux is $F_{\mathrm{vib}}^{i}(\Omega)=-d \Pi_{\mathrm{vib}}^{i} / d t$, where $\Pi_{\mathrm{vib}}^{i}=\rho \operatorname{Aav}^{2} \Lambda_{i}^{2} / \lambda_{i}^{2}-2 \rho \operatorname{Aav}^{2} \Lambda_{i}^{2} / \lambda_{i}^{2} \cos \left(\omega_{i} t\right)$ from Eq. (4) (but with a factor of 2 applied since Eq. (4) is for a half DCB). Substituting these results into Eq. (6) gives the ERR component due to vibration as

$$
\begin{aligned}
G_{\mathrm{vib}} & =-\frac{1}{b} \sum_{i=1}^{\infty}(-1)^{i} f_{i} \frac{d \Pi_{\mathrm{vib}}^{i}}{d a} \\
= & \frac{4 \sqrt{\rho A E I} v^{2} t}{b a^{2}} \sum_{i=1}^{\infty}(-1)^{i} \Lambda_{i}^{2} f_{i} \sin \left(\omega_{i} t\right) \\
& +\frac{2 \rho A v^{2}}{b} \sum_{i=1}^{\infty}(-1)^{i} \frac{\Lambda_{i}^{2}}{\lambda_{i}^{2}} f_{i} \cos \left(\omega_{i} t\right)-\frac{\rho A v^{2}}{b} \sum_{i=1}^{\infty}(-1)^{i} \frac{\Lambda_{i}^{2}}{\lambda_{i}^{2}} f_{i} .
\end{aligned}
$$


The ratio $f_{i}=C_{\mathrm{p}}^{1} / C_{\mathrm{g}}^{i}$ relates the energy flux to the dispersion of flexural waves in beams. If the beam is considered as a waveguide with energy input at one end and transmitted along the beam, then the total energy received at the other end of the beam should be modified by $f_{i}$ for the $i$ th vibration mode because of dispersion. The ratio $f_{i}$ is therefore a reduction factor that characterizes the energy transmission ability of beams as waveguides to generate the ERR. It is an inherent property of beams with a given set of boundary conditions. The phase speed of the first mode flexural wave is $C_{\mathrm{p}}^{1}=\sqrt{\omega_{1}} \sqrt[4]{E I /(\rho A)}$ and the group speed of the $i$ th mode flexural wave is $C_{\mathrm{g}}^{i}=2 \sqrt{\omega_{i}} \sqrt[4]{E I /(\rho A)}[20]$, giving $f_{i}=\sqrt{\omega_{1} /\left(4 \omega_{i}\right)}=\lambda_{1} /\left(2 \lambda_{i}\right)$.

For a stationary delamination in a DCB the dynamic ERR is therefore

$$
\begin{aligned}
G_{\text {stationary }}^{\text {global }} & =\frac{9 E I v^{2} t^{2}}{b a^{4}}-\frac{33 \rho A v^{2}}{140 b}+\frac{4 \sqrt{\rho A E I} v^{2} t}{b a^{2}} \sum_{i=1}^{\infty}(-1)^{i} \Lambda_{i}^{2} f_{i} \sin \left(\omega_{i} t\right) \\
& +\frac{2 \rho A v^{2}}{b} \sum_{i=1}^{\infty}(-1)^{i} \frac{\Lambda_{i}^{2}}{\lambda_{i}^{2}} f_{i} \cos \left(\omega_{i} t\right)-\frac{\rho A v^{2}}{b} \sum_{i=1}^{\infty}(-1)^{i} \frac{\Lambda_{i}^{2}}{\lambda_{i}^{2}} f_{i} .
\end{aligned}
$$

Note that this ERR expression was derived based on a global energy balance along with a correction for the dispersive properties of beams as waveguides; it is therefore referred to as global ERR in this work.

\subsubsection{Simplified dynamic ERR with vibration}

For a DCB under quasi-static loads, the ERR can be calculated by $G_{\text {static }}=M^{2}(a, t) /(b E I)$ , where $M(a, t)=E I w^{(2)}(a, t)$ is the crack-tip bending moment. The static ERR is a local quantity related to the crack tip since it is only a function of the crack-tip bending moment.

Similarly, for a delamination propagating in a DCB under dynamic loading, Freund [18] derived the ERR using a crack-tip energy flux integral as

$$
G_{\text {propagation }}^{\text {local }}=\frac{M^{2}(a, t)}{b E I}\left(1-\frac{\dot{a}^{2}}{C_{0}^{2}}\right),
$$

where $C_{0}$ is the longitudinal wave speed with $C_{0}^{2}=E / \rho$. The dynamic ERR of a propagating delamination is only a function of the delamination propagation speed $\dot{a}$ and the crack-tip bending moment.

Since the ERR under quasi-static loads and the ERR of a propagating delamination under dynamic loads can both be determined by the crack-tip local quantities, it is an interesting question whether the dynamic ERR of a stationary delamination can be determined by setting 
$\dot{a}=0$ in Eq. (10) and using the vibrational deflection in Eq. (1) to determine $M(a, t)$. This would give a simplified solution for dynamic ERR for a stationary delamination compared to Eq. (9).

It should be recognized that Eq. (1) is a vibrational solution, which assumes adequate time for all the flexural waves to form standing waves. Eq. (10), however, considers the actual values of the crack-tip quantities at a given time. This means that the stationary dynamic ERR calculated with Eqs. (1) and (10) together can only become accurate after a certain period of time after $t=0$, at least after the establishment of all the standing waves. Furthermore, the calculated ERR is overestimated during this initial period since the calculation assumes that the energies of all flexural waves are immediately available at crack tip, when, in fact, flexural waves need time to travel along the beam (with lower-frequency ones travelling more slowly due to the dispersive property of flexural waves in 1D waveguides). By comparison, the global ERR in Eq. (9) accounts for wave dispersion (by considering the energy flux into a contour around the crack tip), even though it is also based on the vibrational deflection of Eq. (1).

By combining Eqs. (1) and (10) and setting $\dot{a}=0$, the simplified dynamic ERR for a stationary delamination of a DCB under dynamic loads is

$$
G_{\text {stationary }}^{\text {local }}=\frac{9 E I v^{2} t^{2}}{b a^{4}}+\frac{12 \sqrt{\rho A E I} v^{2} t}{b a^{2}} \sum_{i=1}^{\infty} \frac{\Lambda_{i}}{\lambda_{i}} \sin \left(\omega_{i} t\right)+\frac{4 \rho A v^{2}}{b}\left[\sum_{i=1}^{\infty} \frac{\Lambda_{i}}{\lambda_{i}} \sin \left(\omega_{i} t\right)\right]^{2} .
$$

It is referred to as local ERR in this work since it is determined by the crack-tip bending moment only. It consists of three components: the first term is the ERR component due to quasi-static motion, the second term is the ERR component due to coupling of local vibration and quasistatic motion, and the third term is the ERR component due to local vibration.

To determine under what conditions Eq. (11) is applicable and potentially equivalent to Eq. (9), a relative ERR difference between the local and global methods is defined as $\Delta G / G_{\text {st }}^{\mathrm{U}}=\left(G_{\text {stationary }}^{\text {local }}-G_{\text {stationary }}^{\text {global }}\right) / G_{\text {st }}^{\mathrm{U}}$. It is the difference between the ERR from the two methods, divided by $G_{\mathrm{st}}^{\mathrm{U}}=9 E I v^{2} t^{2} /\left(b a^{4}\right)$ (the ERR component due to strain energy of quasistatic motion, or equivalently, the static ERR without any dynamic effect). Both methods give the same $G_{\mathrm{st}}^{\mathrm{U}}$, which makes it an appropriate choice as normalization factor when defining the relative ERR difference.

To study the characteristics of this relative difference, a relative time scale $n$ is also defined: $n=t / \tau=\lambda_{1} /\left(a^{2} t\right) \sqrt{E I /(\rho A)}$. It is the time $t$ divided by the time taken for the first 
mode flexural wave to travel the delamination length $a$. The phase speed of the first mode flexural wave is $C_{\mathrm{p}}^{1}=\lambda_{1} \sqrt{E I /(\rho A)} / a$ and the time for this wave to travel from the excitation point (i.e. the free end) to the crack tip is $\tau=a / C_{\mathrm{p}}^{1}=a^{2} /\left(\lambda_{1} \sqrt{E I /(\rho A)}\right)$.

Based on Eqs. (9) and (11), the relative ERR difference between two methods simplifies to

$$
\begin{aligned}
\frac{\Delta G}{G_{\mathrm{st}}^{\mathrm{U}}} & =\frac{2}{9} \frac{\lambda_{1}}{n} \sum_{i=1}^{\infty}\left[\frac{6 \Lambda_{i}}{\lambda_{i}}-(-1)^{i} \Lambda_{i}^{2} \frac{\lambda_{1}}{\lambda_{i}}\right] \sin \left(\lambda_{i} \frac{n}{\lambda_{1}}\right) \\
& +\frac{1}{18} \frac{\lambda_{1}^{2}}{n^{2}} \sum_{i=1}^{\infty}(-1)^{i} \frac{\lambda_{1} \Lambda_{i}^{2}}{\lambda_{i}^{3}}\left[1-2 \cos \left(\lambda_{i} \frac{n}{\lambda_{1}}\right)\right] \\
& +\frac{4}{9} \frac{\lambda_{1}^{2}}{n^{2}}\left[\sum_{i=1}^{\infty} \frac{\Lambda_{i}}{\lambda_{i}} \sin \left(\lambda_{i} \frac{n}{\lambda_{1}}\right)\right]^{2}+\frac{11}{420} \frac{\lambda_{1}^{2}}{n^{2}}
\end{aligned}
$$

Eq. (12) is dimensionless and universal for DCBs. Evolution of the relative ERR difference $\Delta G / G_{\mathrm{st}}^{\mathrm{U}}$ with the relative time $n$ is shown in Fig. 3a based on the first ten vibration modes. Fig. $3 b$ shows the same data, but with the $y$ axis transformed to enhance low-amplitude variations of the relative ERR difference for both positive and negative values.

(a)

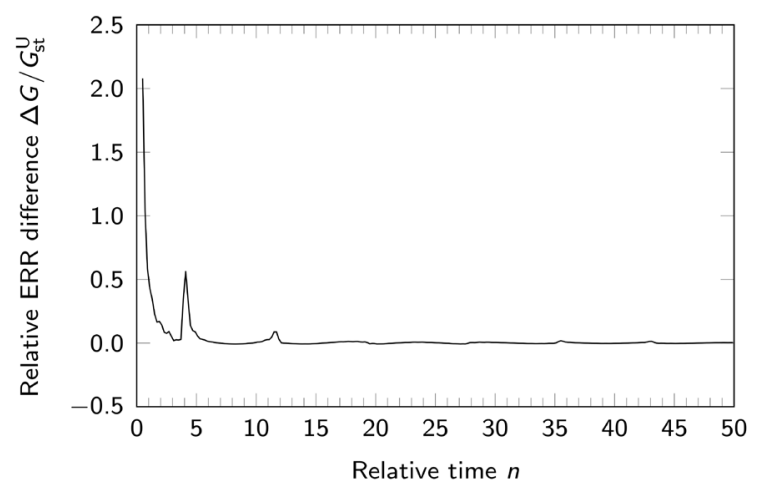

(b)

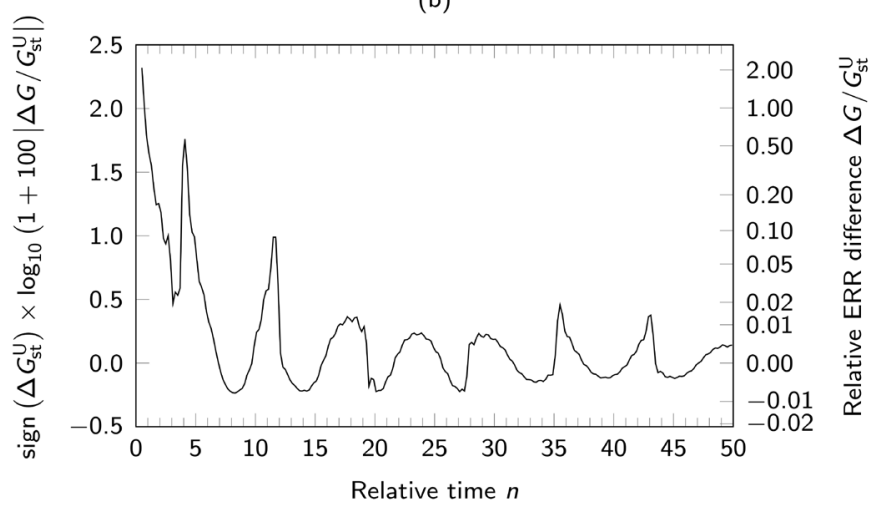

Fig. 3. Evolution of the relative ERR difference between local and global methods with $n$

Apparently the relative ERR difference is mainly positive (Fig. 3). This indicates that the local ERR is larger than the global ERR, consistent with the discussion above. The most significant overestimation happens in the range $0<n<5$; for $n>5$, it rapidly decreases to close to zero. For $n>12$, the relative ERR difference is within approximately $-0.7 \%<\Delta G / G_{\mathrm{st}}^{\mathrm{U}}<1.9 \%$. The global and local ERR solutions can therefore be considered as equivalent for predicting the dynamic ERR provided that enough time has passed, with $0 \leq n \leq 12$ being the transition period for vibrational solution to be applicable. Note that when 
$n=2$, the first mode flexural wave returns to the free end, with all the standing waves already established. The local ERR solution, however, still needs time to 'even out' the additional 'artificial' energy in comparison to the global ERR solution, explaining the duration of the transition period beyond $n=2$.

One further simplification can be made to Eq. (11): since $\left[\sum_{i=1}^{\infty} \Lambda_{i} / \lambda_{i} \sin \left(\omega_{i} t\right)\right]^{2} \leq\left[\sum_{i=1}^{\infty} \Lambda_{i} / \lambda_{i}\right]^{2}$, with the values of $\Lambda_{i}$ and $\lambda_{i}$ given in Appendix A, the third term of Eq. (11) can be no larger than $0.2295 \rho A v^{2} / b$. This third term is therefore small in comparison to the second term of Eq. (11) when $n>12$, in which case the dynamic ERR further simplifies to

$$
G_{\text {stationary }}^{\text {local }}=\frac{9 E I v^{2} t^{2}}{b a^{4}}+\frac{12 \sqrt{\rho A E I} v^{2} t}{b a^{2}} \sum_{i=1}^{\infty} \frac{\Lambda_{i}}{\lambda_{i}} \sin \left(\omega_{i} t\right) .
$$

Eq. (13) provides the simplified dynamic ERR based on the local bending moment and vibrational deflection. When $n>12$, it is equivalent to Eq. (9), but for an accurate calculation of ERR when $n<12$, it is advisable to use Eq. (9) instead, which accounts for wave dispersion.

\subsection{Propagating delamination}

\subsubsection{Dynamic ERR for propagating delamination}

For a delamination propagating in a DCB made of a material of moderate density (i.e. the density is not so great that inertia effects cause the delamination surfaces to close and delamination arrest) and constant interface fracture toughness, Chen et al. [15] demonstrated by setting $G=G_{\mathrm{c}}$ that the delamination length and delamination propagation speed are $a(t)=a_{0}+A_{1} \sqrt{t}\left(A_{1}\right.$ is determined by the initial condition) and $\dot{a}=\left(a-a_{0}\right) /\left[2\left(t-t_{0}\right)\right]$, respectively. These solutions, however, do not apply when the DCB has interface fracture toughness that depends on the delamination propagation speed.

For a DCB with delamination-propagation-speed-dependent interface fracture toughness, Freund's formula in Eq. (10) can be applied to determine the dynamic ERR; however, it depends on the crack-tip bending moment. This necessitates solving partial differential equations with a moving boundary condition at the crack tip. This cannot be achieved by rigorous mathematics due to the unknown delamination-length function $a(t)$ [21][22][23]; however, an analytical engineering solution is now developed. 
For a propagating delamination of instantaneous length $a_{1}$, by assuming the deflection given in Eq. (1) holds, the bending moment at the crack tip is $M\left(a_{1}, t\right)=E I w^{(2)}\left(a_{1}, t\right)$. Then, by combining Eqs. (1) and (10) and employing the simplification described in Section 2.1.2, the dynamic ERR is

$$
G_{\text {propagation }}^{\text {local }}=\frac{9 E I v^{2} t^{2}}{b a^{4}}\left(1-\frac{\dot{a}_{1}^{2}}{C_{0}^{2}}\right)+\frac{12 \sqrt{\rho A E I} v^{2} t}{b a_{1}^{2}}\left(1-\frac{\dot{a}_{1}^{2}}{C_{0}^{2}}\right) \sum_{i=1}^{\infty} \frac{\Lambda_{i}}{\lambda_{i}} \sin \left(\omega_{i} t\right) .
$$

The first term is due to quasi-static motion and, therefore, does not need to be adjusted for a propagating delamination. The second term, however, is related to vibration and so does need to be adjusted to account for dispersion and the Doppler effect [24].

By combining Eqs. (6) and (14) for a delamination length of $a_{1}$, the vibration energy flux into a contour around the crack tip is

$$
F_{1}(\Omega)=\dot{a}_{1} b \sum_{i=1}^{\infty} G_{\mathrm{vib}}^{i}\left(a_{1}\right),
$$

where $G_{\text {vib }}^{i}\left(a_{1}\right)=12 \sqrt{\rho A E I} v^{2} t\left(C_{0}^{2}-\dot{a}_{1}^{2}\right) \Lambda_{i} \sin \left(\omega_{i} t\right) /\left(\lambda_{i} b a_{1}^{2} C_{0}^{2}\right)$.

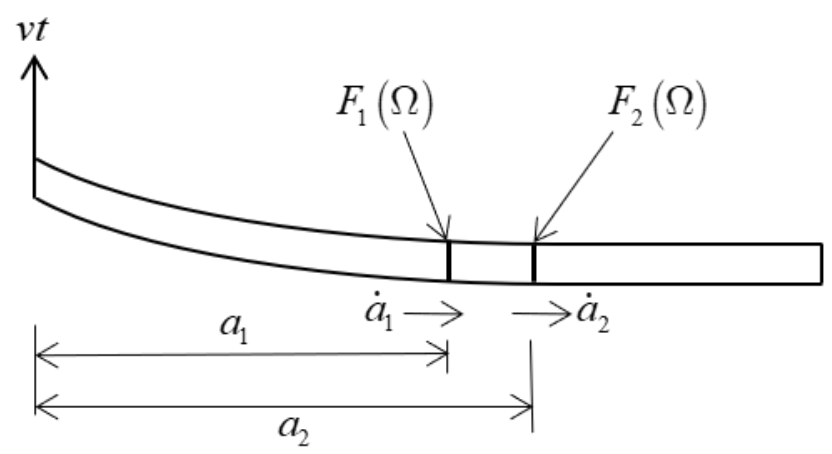

Fig. 4. Delamination propagation from $a_{1}$ to $a_{2}$ over time interval $\Delta t$

Now consider delamination propagation from $a_{1}$ to $a_{2}$ over a time interval $\Delta t$. Ahead of the initial crack-tip position at $x=a_{1}$, a new beam section of length $\left(a_{2}-a_{1}\right)$ is formed as shown in Fig. 4, with the total energy supplied to the new beam section being $F_{1}(\Omega) \Delta t$. Following the same arguments and techniques developed for consideration of dispersion in Section 2.1.1, the energy flux due to $F_{1}(\Omega) \Delta t$ at the new crack tip position at $x=a_{2}$ is

$$
F_{2}(\Omega)=\dot{a}_{1} b \sum_{i=1}^{\infty} f_{i}^{\prime} G_{\mathrm{vib}}^{i}\left(a_{1}\right)
$$


where $f_{i}^{\prime}$ is the reduction factor that accounts for the dispersion of flexural waves in the new beam section. It is a function of the new beam section's boundary conditions only.

By combining Eqs. (6) and (16), the ERR component due to vibration at the new crack length of $a_{2}$ is therefore

$$
G_{\text {vib }}\left(a_{2}\right)=\frac{F_{2}(\Omega)}{b \dot{a}_{2}}=\frac{12 \sqrt{\rho A E I} v^{2} t}{b a_{1}^{2}} \frac{\dot{a}_{1}}{\dot{a}_{2}}\left(1-\frac{\dot{a}_{1}^{2}}{C_{0}^{2}}\right) \sum_{i=1}^{\infty} \frac{\Lambda_{i}}{\lambda_{i}} f_{i}^{\prime} \sin \left(\omega_{i} t\right) .
$$

The ratio $f_{i}^{\prime}$ is determined in the same way as $f_{i}$ in Section 2.1.1. Note that since the boundary conditions for the new beam section here, formed by delamination propagation, are different to the boundary conditions for the stationary delamination in Section 2.1.1, $f_{i}^{\prime}$ must be different to $f_{i}$. If $\left(a_{2}-a_{1}\right)$ is small then $\dot{a}_{1} \approx \dot{a}_{2}$ and the boundary conditions for the new beam section can be taken as approximately fixed-fixed, where the deflection and slope at both $x=a_{1}$ and $x=a_{2}$ are all zero. From Section 2.1.1 we still have $f_{i}^{\prime}=\lambda_{1}^{\prime} /\left(2 \lambda_{i}^{\prime}\right)$, but now $\lambda_{i}^{\prime}$ is determined from the frequency equation of a beam with fixed-fixed boundary conditions, that is, $\cos \left(\lambda_{i}^{\prime}\right) \cosh \left(\lambda_{i}^{\prime}\right)-1=0$. The values of $\lambda_{i}^{\prime}$ are tabulated in Appendix B.

The frequency of the $i$ th mode flexural wave is $\omega_{i}=\left(\lambda_{i}^{2} / a^{2}\right) \sqrt{E I /(\rho A)}$. These frequencies need to be modified due to the Doppler effect [24]. The frequencies of flexural waves observed at the crack tip decrease with increasing delamination propagation speeds. For a propagating delamination, the actual frequency of the $i$ th-mode flexural wave observed at crack tip is

$$
\omega_{i}^{\prime}=\left(1-\frac{\dot{a}}{C_{\mathrm{p}}^{i}}\right) \omega_{i}
$$

Combining Eqs. (14), (17) and (18), the total dynamic ERR for a propagating crack is

$$
G_{\text {propagation }}^{\text {local }}=\frac{9 E I v^{2} t^{2}}{b a^{4}}\left(1-\frac{\dot{a}^{2}}{C_{0}^{2}}\right)+\frac{12 \sqrt{\rho A E I} v^{2} t}{b a^{2}}\left(1-\frac{\dot{a}^{2}}{C_{0}^{2}}\right) \sum_{i=1}^{\infty} \frac{\Lambda_{i}}{\lambda_{i}} f_{i}^{\prime} \sin \left[\left(1-\frac{\dot{a}}{C_{\mathrm{p}}^{i}}\right) \omega_{i} t\right] \text {. }
$$

\subsubsection{Limiting speed of delamination propagation}

For the first term of Eq. (19), which is the ERR component due to the strain energy of quasistatic motion, the delamination propagation speed $\dot{a}$ should be no greater than $C_{0}=\sqrt{E / \rho}$. This component of ERR would otherwise become negative, inhibiting delamination propagation. For the second term of Eq. (19), which is the ERR component due to vibration, 
there is another constraint in addition to $\dot{a} \leq C_{0}$ : the delamination propagation speed $\dot{a}$ must be no greater than $C_{\mathrm{p}}^{1}$, that is, $\dot{a} \leq C_{\mathrm{p}}^{1}$. Vibration energy would otherwise not be supplied to the propagating crack tip (recall that $C_{\mathrm{p}}^{1}$ is the phase speed of the first mode flexural wave, which carries all the other higher mode waves). This speed is

$$
C_{\mathrm{p}}^{1}=\frac{\lambda_{1}}{a} \sqrt{\frac{E I}{\rho A}}=\frac{\lambda_{1}}{\sqrt{12}} r \sqrt{\frac{E}{\rho}},
$$

where $r=h / a$ is the aspect ratio of the half $\mathrm{DCB}$, and so the limiting speed of delamination propagation in DCBs is proportional to $r$.

Freund [18] derived that for mode I fracture, the crack propagation speed cannot surpass the Rayleigh wave speed $C_{\mathrm{R}}$, where $C_{\mathrm{R}}$ is approximately $C_{\mathrm{R}}=(0.862+1.14 v) C_{\mathrm{S}} /(1+v)$ and the shear wave speed $C_{\mathrm{S}}$ is $C_{\mathrm{S}}=\sqrt{E /[2 \rho(1+v)]}$. The ratio of these two limiting speeds, $C_{\mathrm{p}}^{1}$ and $C_{\mathrm{R}}$, is

$$
\frac{C_{\mathrm{p}}^{1}}{C_{\mathrm{R}}}=\frac{\lambda_{\mathrm{l}}}{\sqrt{6}} \frac{1+v}{(0.862+1.14 v) \sqrt{1-v}} r .
$$

These two different limiting speeds, $C_{\mathrm{p}}^{1}$ and $C_{\mathrm{R}}$, are not in contradiction to each other: instead, $C_{\mathrm{p}}^{1}$ is a development of $C_{\mathrm{R}}$ when applied to a DCB with a given aspect ratio. Freund's original derivation of the limiting crack propagation speed as the Rayleigh wave speed $C_{\mathrm{R}}$ was based on a crack in an infinite sheet. The crack provides a traction-free surface on a semiinfinite medium, where Rayleigh waves can form. If, however, additional structural constraints are included, for example, in form of traction-free surface imposed parallel to the existing one, the semi-infinite medium develops into a plate. For a thick plate, Rayleigh waves become Rayleigh-Lamb waves, which are the superposition of A0 (asymmetric mode) and S0 (symmetric mode) Lamb waves [25]. A0 Lamb waves resemble flexural waves and S0 Lamb waves resembles axial waves [26]. For a thin plate, A0 Lamb waves become the 2D counterpart of flexural waves in Euler-Bernoulli beams [20], and, if axial motion is absent, the influence of S0 Lamb waves can be ignored.

For the increasingly constrained structure described above, as it develops from a semiinfinite sheet to an Euler-Bernoulli beam, Rayleigh waves develop into flexural waves in a beam, and accordingly, the Rayleigh wave speed drops to the phase speed of flexural waves. 
This leads to a reduction in the limiting speed of delamination propagation, as determined by Eq. (21).

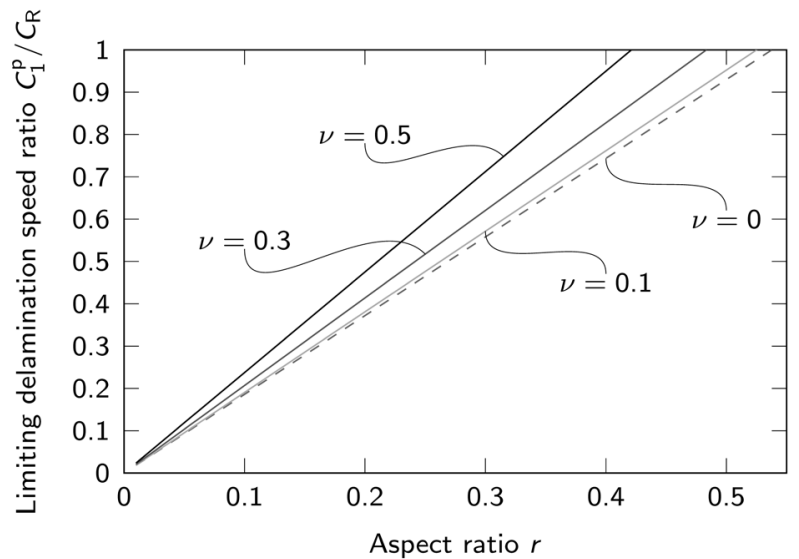

Fig. 5. Effect of aspect ratio on limiting delamination propagation speed for various Poisson's ratios

According to the assessment in Eq. (21), the limiting delamination propagation speed decreases with decreasing aspect ratio and decreasing Poisson's ratio as shown in Fig. 5. The limiting speed of delamination in a DCB therefore decreases with the increasing delamination length. For the conventional DCB test employed to determine the mode-I delamination toughness in fiber-reinforced polymer-matrix composites, the aspect ratio magnitude typically ranges from 0.01 to 0.1 [1], and so, from Eq. (21), the corresponding limiting speed of delamination propagation is therefore in the range $0.02 C_{\mathrm{R}}$ to $0.25 C_{\mathrm{R}}$.

There are currently relatively few experimental data on dynamic delamination propagation available in the literature [11], at least partly due to the challenges such tests pose for experimental-apparatus design. Experimental data for high loading rates are available in Refs. [6][7], which used a servo-hydraulic test machine with a 'lost motion device' to achieve opening rates up to $15 \mathrm{~m} \mathrm{~s}^{-1}$, and in Ref. [27], which used an electromagnetic Hopkinson bar to obtain opening rates up to $30 \mathrm{~m} \mathrm{~s}^{-1}$. 

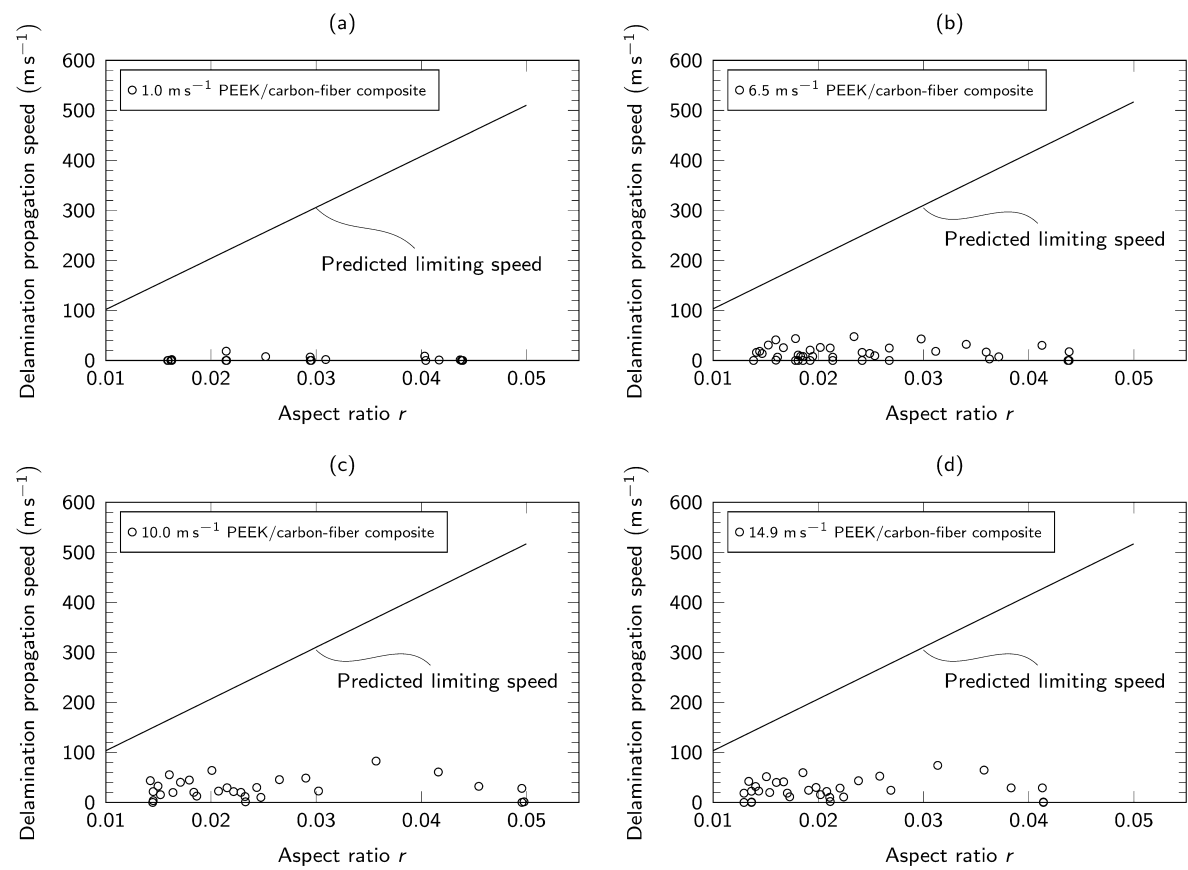

(e)

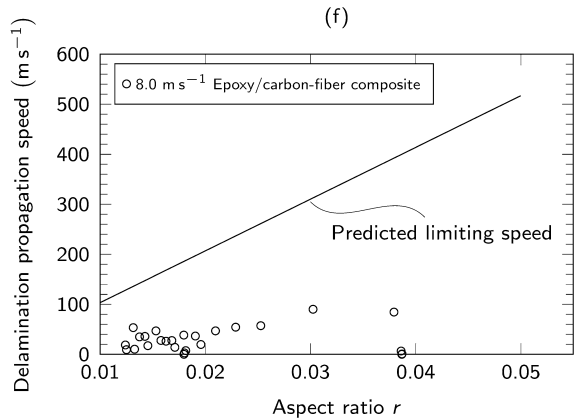

(g)

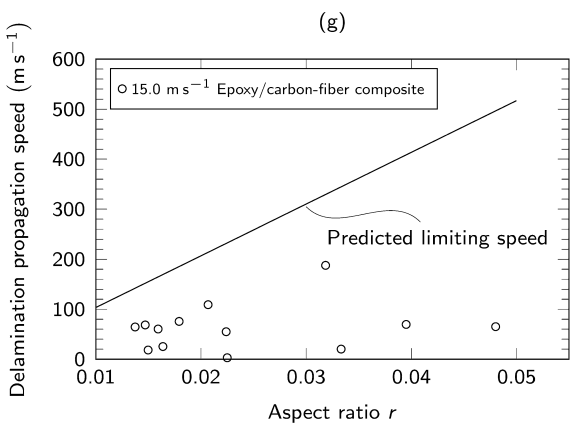

Fig. 6. Comparison of experimentally measured delamination propagation speeds under various opening (loading) rates [6][7] against theoretically predicted limiting speed

The delamination propagation speeds measured in Refs. [6][7] under various loading rates for two kinds of fiber-reinforced polymer matrix composite-PEEK/carbon-fiber and epoxy/carbon-fiber-are plotted in Fig. 6. The theoretical limiting speed of delamination propagation, predicted by Eq. (20), is also plotted. The measured delamination propagation speeds are all well below the predicted limiting speed for all the opening rates (up to $15 \mathrm{~m} \mathrm{~s}^{-1}$ ). 
Ref. [27] reports delamination propagation speeds for 18 specimens of uniaxial carbon/epoxy composite, ranging from $108 \mathrm{~m} \mathrm{~s}^{-1}$ to $253 \mathrm{~m} \mathrm{~s}^{-1}$. For the given opening rates of up to $30 \mathrm{~m} \mathrm{~s}^{-1}$, the lowest predicted limiting speed of delamination propagation from Eq. (20) is $385 \mathrm{~m} \mathrm{~s}^{-1}$, and so the measured delamination propagation speeds are also within the predicted limit.

A further two points should be noted concerning Eq. (21): First, for aspect ratios in the range 0.4-0.5 when the thickness and delamination length are of the same order, it might appear that the limiting delamination propagating speed can reach $C_{\mathrm{R}}$ as Rayleigh waves can form [28]. The Euler-Bernoulli beam assumption, however, requires that $h / a<0.1$. Second, when the aspect ratio approaches zero, it might appear that the limiting delamination propagation speed should also approach zero, which would mean that delamination cannot propagate. For zero aspect ratio, however, the structure instead behaves like a string, and is unable to bear compression or bending loads. This should not be the case with real DCB configurations.

\section{Experimental and finite-element-method verification}

\subsection{Verification against experiments}

There are relatively few experimental studies of DCBs under high loading rates in the literature [11]. Blackman et al. [6][7], however, performed a comprehensive series of experiments under high opening rates of up to $15 \mathrm{~m} \mathrm{~s}^{-1}$. Two of these DCB experiments, with PEEK/carbon-fiber composite under opening rates of $6.5 \mathrm{~m} \mathrm{~s}^{-1}$ and $10 \mathrm{~m} \mathrm{~s}^{-1}$, were selected to verify the analytical theory developed in Section 2. For this composite, the longitudinal modulus is taken as $115 \mathrm{GPa}$, the Poisson's ratio as 0.28 and the density as $1540 \mathrm{~kg} \mathrm{~m}^{-3}$ [6][7]. The half DCB thickness is $1.5 \mathrm{~mm}$, and the width is $20 \mathrm{~mm}$. A plane strain condition is therefore assumed in the analytical calculations with the effective Young's modulus taken as $E /\left(1-v^{2}\right)=124.78 \mathrm{GPa}$.

Before delamination propagation, that is, for stationary delamination, Eq. (9) was used, which provides a continuous ERR with respect to time since the delamination length does not increase. During delamination propagation, Eq. (19) was used. The first five vibration modes were considered in both cases, which Ref. [16] showed to be sufficient.

The original experimental data of delamination length versus time [6][7] includes 51 data points at evenly spaced intervals for the test with the $6.5 \mathrm{~m} \mathrm{~s}^{-1}$ opening rate, and 31 points for the test with the $10 \mathrm{~m} \mathrm{~s}^{-1}$ opening rate. A central difference calculation was therefore used to 
estimate the delamination propagation speed with $\dot{a}\left(t_{i}\right)=\left[a\left(t_{i+1}\right)-a\left(t_{i-1}\right)\right] /\left(t_{i+1}-t_{i-1}\right)$ since there are not enough data points to get an accurate estimate otherwise. When calculating the ERR, an effective delamination length of $a_{\text {eff }}=(a+\Delta)$ was used, where $a$ is the actual delamination length and $\Delta$ is an additional experimentally-determined delamination length added to compensate for crack tip rotation [6][7], which otherwise is not captured by EulerBernoulli beams. The corresponding values of $\Delta$ for each test are from Refs. [6][7], in which $\Delta$ for the $6.5 \mathrm{~m} \mathrm{~s}^{-1}$ opening rate is $4.4 \mathrm{~mm}$. For the $10 \mathrm{~m} \mathrm{~s}^{-1}$ opening rate, $\Delta$ was calculated by linear interpolation between the $6.5 \mathrm{~m} \mathrm{~s}^{-1}$ and $14.9 \mathrm{~m} \mathrm{~s}^{-1}$ opening rates giving $\Delta=5 \mathrm{~mm}$.

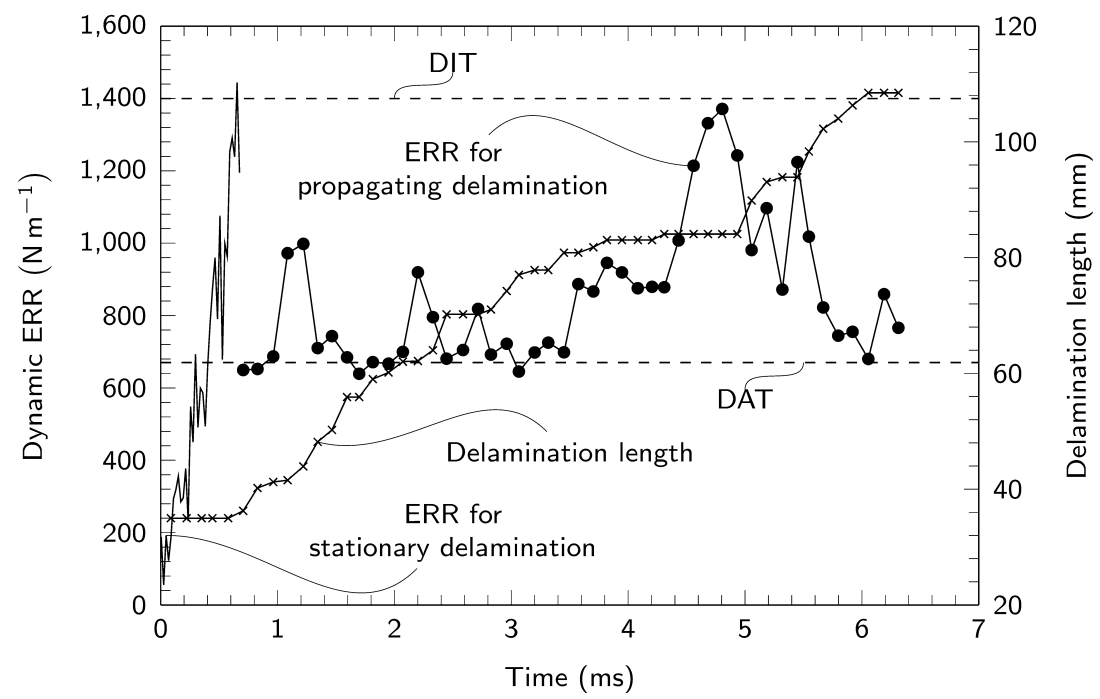

Fig. 7. Evolution of dynamic ERR and delamination length for $6.5 \mathrm{~m} \mathrm{~s}^{-1}$ loading rate based on experimentally observed delamination propagation speed

The theoretical results of dynamic ERR for the DCB test with the $6.5 \mathrm{~m} \mathrm{~s}^{-1}$ opening rate are plotted in Fig. 7. They are based on the experimentally observed delamination length and propagation speeds (the delamination length is also plotted in Fig. 7 with values on the secondary axis). Note that Ref. [9] simulates Blackman et al.'s [6][7] experiments using the FEM, and in these simulations, the delamination initiation time is higher than the actual delamination initiation time in the experiments. This is because "the lost motion device was set to allow a period of pre-travel to ensure that the test was conducted at constant velocity" [6][7], and so this pre-travel period should be taken into consideration. The FEM results in Ref. [9] were therefore used to shift the initiation time in the experimental data. The experimental results in Fig. 7 are plotted on this shifted time scale in order to make a valid comparison with the theory. The DIT of $1400 \mathrm{~N} \mathrm{~m}^{-1}$ and the DAT of $670 \mathrm{~N} \mathrm{~m}^{-1}$ are taken from the same reference 
[9], in which Liu et al. performed a parametric FEM study of different values of DIT and DAT aiming to match the experimental data. These values are not required in the calculation of dynamic ERR and are only shown for comparison.

Using the analytical theory developed in Section 2 and the experimentally observed delamination length and propagation speed, the dynamic ERR was calculated for both stationary delamination and propagation. For stationary delamination, it is expected that the delamination should not propagate until the ERR exceeds the DIT $\left(1400 \mathrm{~N} \mathrm{~m}^{-1}\right)$. For propagating delamination, it is expected that the delamination should propagate if the ERR exceeds the DAT $\left(670 \mathrm{~N} \mathrm{~m}^{-1}\right)$ and should be arrested otherwise. Moreover, once the dynamic ERR drops below the DAT and delamination propagation is arrested, it is expected that it reinitiates (i.e. continue propagating again) only once the dynamic ERR builds up and exceeds the DIT again.

Apparently, the developed analytical theory is generally in excellent agreement with the experimental results (Fig. 7): (1) The delamination initiates and begins to propagate once the dynamic ERR reaches the expected the DIT (i.e. after $0.6 \mathrm{~ms}$ ). (2) The dynamic ERR is equal to or greater than the DAT, while it propagates continuously between about $0.6 \mathrm{~ms}$ and $3.5 \mathrm{~ms}$. Note that a small number of data points in this period are very slightly below the DAT, and that in the period between $2.4 \mathrm{~ms}$ and $2.7 \mathrm{~ms}$ (with three sample points), there appears to be a very short period of arrest. Nevertheless, the general tendency in the period $0.6 \mathrm{~ms}$ to $3.5 \mathrm{~ms}$ is that of propagation with the dynamic ERR equal to or greater than the DAT. There are several considerations which indicate the general tendency is more important than close attention to singular or small groups of data points when interpreting these experimental results. In both cases, at least part of the cause is the relatively few experimental sample points, which prevented accurate estimation of the delamination propagation speed. Also, concerning the short arrest period, the FEM simulation shows that there is no such small arrest period (this is discussed in Section 3.2 and shown in Fig. 9). In addition, dynamic tests are well-known to be probabilistic in nature [29], particularly where damage is concerned and on small scales of time and space, whereas the developed theory is deterministic. For all these reasons, it is sensible to consider that the delamination propagates during the short period between $2.4 \mathrm{~ms}$ and $2.7 \mathrm{~ms}$ with the dynamic ERR equal to or greater than the DAT (thus permitting continued propagation). (3) At about $3.5 \mathrm{~ms}$, for the reasons described above, it is also within the probable error margin that the dynamic ERR drops below the DAT, and the propagation is arrested. (4) Once propagation is arrested, the dynamic ERR needs to exceed the DIT again in order to re-initiate. Indeed, there is a delamination arrest period between $3.5 \mathrm{~ms}$ and about $4.8 \mathrm{~ms}$, 
where the dynamic ERR is lower than the DIT. (5) At about $4.8 \mathrm{~ms}$, the dynamic ERR reaches the DIT again and then the delamination propagates until about $6 \mathrm{~ms}$ with the dynamic ERR within the limits set by the DIT and the DAT. (6) At $6 \mathrm{~ms}$, the dynamic ERR drops below the DAT and the propagation arrests once more.

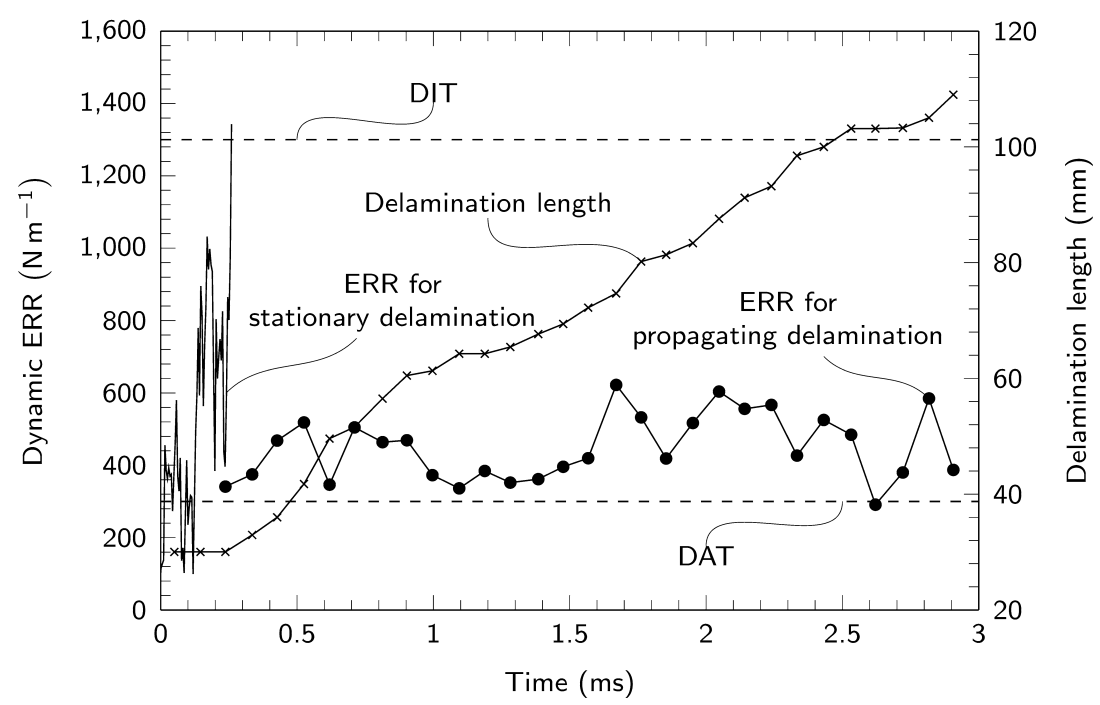

Fig. 8. Evolution of dynamic ERR and delamination length for $10 \mathrm{~m} \mathrm{~s}^{-1}$ loading rate based on experimentally observed delamination propagation speed

The theoretical results of dynamic ERR for the DCB test with the $10 \mathrm{~m} \mathrm{~s}^{-1}$ opening rate are presented in Fig. 8. These results are based on the experimentally observed delamination length and propagation speeds (the delamination length is also plotted in Fig. 8 with values on the secondary axis). As explained above, to account for the period of pre-travel in the experiment, the experimental results are plotted on a shifted time scale, with the required amount of shift determined using the FEM results in Ref. [9]. The values of DIT and DAT were also determined in Ref. [9] as $1300 \mathrm{~N} \mathrm{~m}^{-1}$ and $300 \mathrm{~N} \mathrm{~m}^{-1}$, respectively. These toughness values are not required in the calculation of dynamic ERR and are only shown for comparison.

Fig. 8 again demonstrates an excellent agreement between the developed analytical theory and the experimental results: (1) The delamination initiates and begins to propagate once the dynamic ERR reaches the expected DIT. (2) After crack initiation, there is a significant drop of dynamic ERR, which remains above the DAT until $t=2.6 \mathrm{~ms}$, when the propagation arrests.

In both comparisons with dynamic DCB experiment at different opening rates, the developed analytical theory predicts dynamic ERRs that are in line with the observed delamination propagation behavior. This is a strong confirmation that the developed analytical 
theory accurately predicts the values of DIT and DAT at times of the delamination initiation or re-initiation, and arrest.

\subsection{Verification against FEM simulations}

Section 3.1 showed that the developed analytical theory can accurately predict the main features of delamination behaviour such as initiation, propagation and arrest. More detailed comparisons between the developed analytical theory and experiment were not possible, however, since dynamic ERR cannot be measured directly from experiments without postprocessing (for example, using the developed theory), and because of the insufficient number of sample points for accurate measurement of the delamination propagation speed.

Instead, the results from FEM analysis in Ref. [9], which simulated the same experiments considered in Section 3.1 [6][7], were used to further verify the developed analytical theory. The FEM analysis by Liu et al. [9] used interfacial thick level set (ITLS) modelling, which is similar to a cohesive-zone approach, to simulate 3D DCBs under different loading rates. Full details are given in Ref. [9]. Following the same procedure described in Section 3.1 for experiments, the FEM results for delamination propagation speed were used together with the developed analytical theory to determine the analytical dynamic ERR, which could then be compared directly with the respective FEM results.

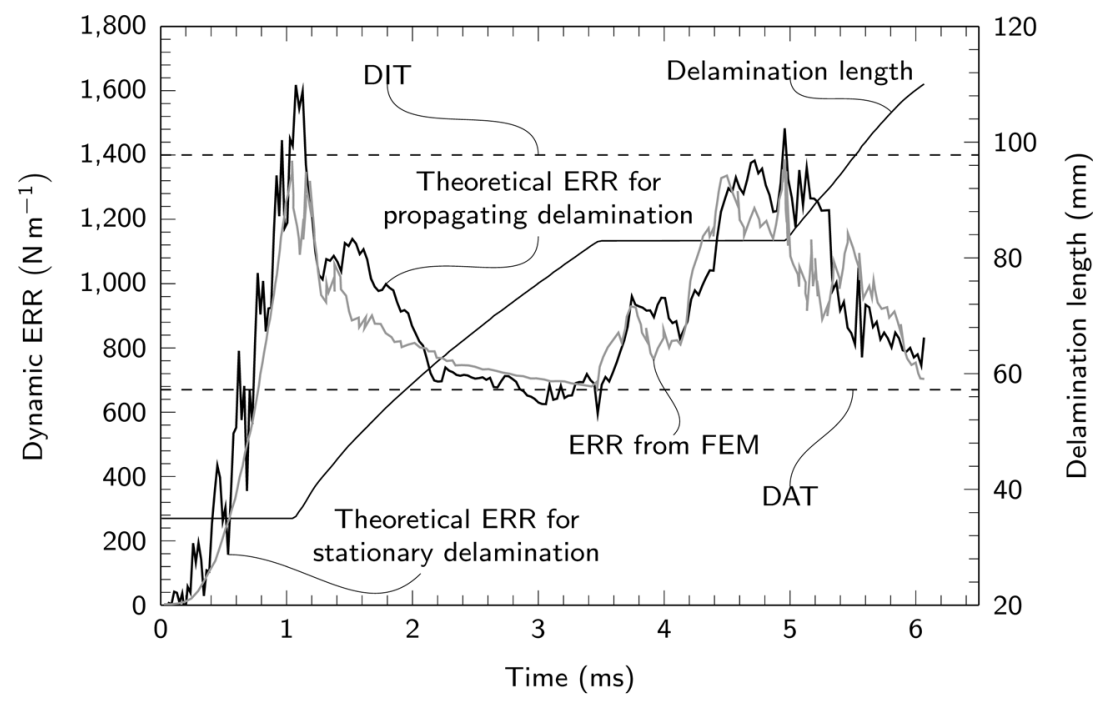

Fig. 9. Evolution of dynamic ERR and delamination length for $6.5 \mathrm{~m} \mathrm{~s}^{-1}$ loading rate based on FEM results of delamination propagation speed

The dynamic ERR calculated with the developed analytical theory (based on the delamination length-time curve obtained from the FEM simulation) and the FEM results for 
the $6.5 \mathrm{~m} \mathrm{~s}^{-1}$ loading case are compared in Fig. 9. They are in excellent agreement for both stationary delamination and propagating delamination. Note that the developed analytical theory predicts a slightly higher ERR in comparison to the FEM. This is reasonable since the FEM model used a 3D formulation with the anisotropic material properties of $E_{1}=115 \mathrm{GPa}$ and $E_{2}=8 \mathrm{GPa}$, making this model less stiff than the analytical one. The discrepancy is not significant.

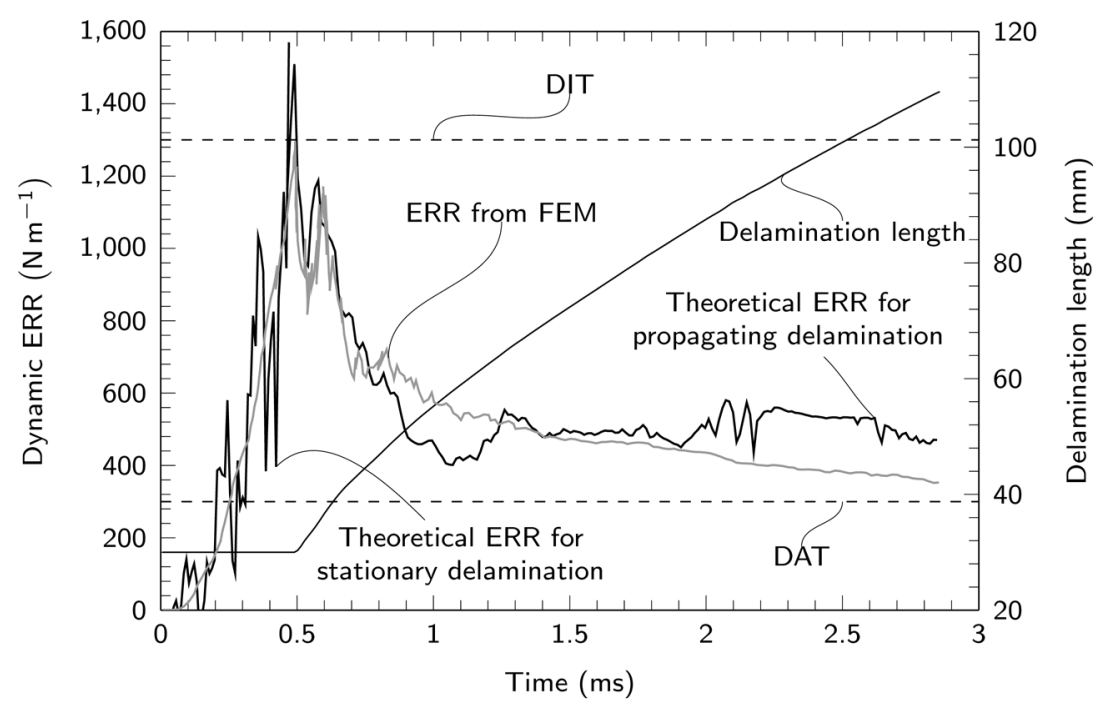

Fig. 10. Evolution of dynamic ERR and delamination length for $10 \mathrm{~m} \mathrm{~s}^{-1}$ loading rate based on FEM results of delamination propagation speed

The dynamic ERR determined with the developed analytical theory (based on the evolution of the delamination length calculated with the FEM result) and the numerical result are compared in Fig. 10 for the $10 \mathrm{~m} \mathrm{~s}^{-1}$ loading case. They are in excellent agreement for both regimes of delamination - stationary and propagating.

The analytical theory developed in Section 2 for both stationary and propagating delamination is therefore verified and can accurately predict the dynamic ERR in DCB tests under high loading rate, and also to characterize the main features of delamination behaviour such as its initiation, propagation and arrest.

\section{Conclusion}

Analytical theory was developed for the dynamic mode-I delamination behavior of a DCB under high loading rate, including both stationary and propagating delamination regimes. Analytical solutions for the dynamic ERR were derived based on the vibration of EulerBernoulli beams taking account of flexural-wave dispersion. 
Two solutions for the ERR of a stationary delamination were given: an accurate one and a simplified one. The accurate solution is based on global energy balance, structural vibration and wave dispersion. The simplified solution is 'local', since it is based on the crack-tip bending moment. For the simplified solution to be accurate, it was shown that enough time $t$ should pass to allow the establishment of all the standing waves, with relative time $n>12$, where $n=t C_{\mathrm{p}}^{1} / a, C_{\mathrm{p}}^{1}$ is the phase speed of first mode flexural wave and $a$ is the delamination length.

For a propagating delamination, a solution for the dynamic ERR was derived using the same simplification with the crack tip bending moment, also accounting for dispersion and the Doppler effect at the crack-tip. The developed theory was used to determine the limiting speed of delamination propagation in DCBs. For a conventional DCB test with a magnitude of aspect ratio $h / a$ typically ranging from 0.01 to 0.1 (where $h$ is the DCB's arm thickness), the limiting speed of delamination propagation was determined to be in the range $0.02 C_{\mathrm{R}}$ to $0.25 C_{\mathrm{R}}$, where $C_{\mathrm{R}}$ is the Rayleigh wave speed.

The developed analytical theory was verified against both experimental data and results of FEM simulations. It accurately predicted the dynamic ERR in comparison to FEM simulations; and it provided correct assessment of delamination behavior, including delamination initiation, arrest and re-initiation observed in the experiments. The theory can therefore be used to determine the delamination initiation toughness, delamination arrest toughness and delamination re-initiation toughness.

Accurately determining the dynamic ERR under high loading rate is essential for properly understanding of dynamic delamination behavior, determining the dynamic delamination toughness of a studied material and for predicting the extent of delamination. To the authors' knowledge, this has not yet been achieved by analytical theory with the consideration of vibration, but only by computationally intensive numerical simulations. So, the developed theory is of great value for understanding the delamination behavior of layered materials and can be readily applied in many engineering applications. One valuable application of the developed theory is to determine a material's dynamic loading-rate-dependent delamination toughness by providing the analytical theory to post-process test results of dynamic DCB delamination. 


\section{Appendices}

Appendix A. Derivation of deflection solution

Consider the beam shown in Fig. A1. By introducing a shifting function $F(x)$ [30], the deflection is of the following form:

$$
w(x, t)=w_{\mathrm{fv}}(x, t)+F(x) v t .
$$

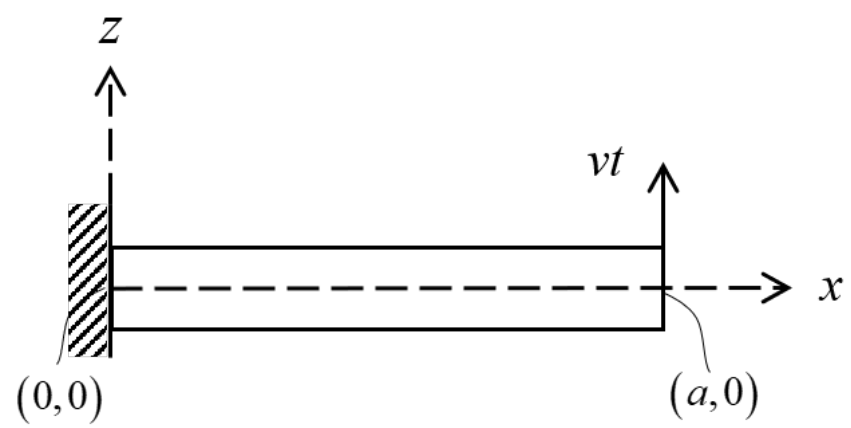

Fig. A1 Beam with effective boundary condition representing one DCB arm

By combining Eq. (A1) and the equation of motion [31] of $E I w^{(4)}(x, t)+\rho A \ddot{w}(x, t)=0$, and forcing homogeneous condition, two differential equations are obtained as follows for the freevibration component $w_{\mathrm{fv}}(x, t)$ and shifting function $F(x)$, respectively:

$$
\begin{gathered}
E I w_{\mathrm{fv}}^{(4)}(x, t)+\rho A \ddot{w}_{\mathrm{fv}}(x, t)=0, \\
F^{(4)}(x)=0 .
\end{gathered}
$$

The solution of the shifting function $F(x)$ is found to be

$$
F(x)=\left(\frac{3 x^{2}}{2 a^{2}}-\frac{x^{3}}{2 a^{3}}\right)
$$

The general solution of the free-vibration component $w_{\mathrm{fv}}(x, t)$ is found by separation of variables as

$$
w_{\mathrm{fv}}(x, t)=\sum_{i=1}^{\infty} W_{i}(x)\left[T_{i}(0) \cos \left(\omega_{i} t\right)+\frac{\dot{T}_{i}(0)}{\omega_{i}} \sin \left(\omega_{i} t\right)\right],
$$

where $W_{i}(x)$ is the $i$ th normal mode, which is solved to be $W_{i}(x)=(\rho A a)^{-1 / 2} \phi_{i}(x)$, with $\phi_{i}(x)$ being the shape function, 
$\phi_{i}(x)=\cosh \left(\frac{\lambda_{i}}{a} x\right)-\cos \left(\frac{\lambda_{i}}{a} x\right)-\sigma_{i}\left[\sinh \left(\frac{\lambda_{i}}{a} x\right)-\sin \left(\frac{\lambda_{i}}{a} x\right)\right] . T_{i}(0)$ and $\dot{T}_{i}(0)$ are the initial modal displacement and modal velocity, respectively. $\lambda_{i}$ is determined by the frequency equation, $\tan \left(\lambda_{i}\right)-\tanh \left(\lambda_{i}\right)=0$, and $\sigma_{i}=\left[\cosh \left(\lambda_{i}\right)-\cos \left(\lambda_{i}\right)\right] /\left[\sinh \left(\lambda_{i}\right)-\sin \left(\lambda_{i}\right)\right]$.

The solution for the initial modal displacement and modal velocity are, according to Ref. [31],

$$
\begin{aligned}
& T_{i}(0)=\int_{0}^{a} \rho A W_{i}(x) w_{\mathrm{fv}}(x, 0) d x, \\
& \dot{T}_{i}(0)=\int_{0}^{a} \rho A W_{i}(x) \dot{w}_{\mathrm{fv}}(x, 0) d x .
\end{aligned}
$$

By using Eq. (A1) and forcing homogeneous conditions, the initial conditions for the freevibration component are $w_{\mathrm{fv}}(x, 0)=0$ and $\dot{w}_{\mathrm{fv}}(x, 0)=-F(x) v$. The $i$ th modal displacement is therefore zero, that is, $T_{i}(0)=0$, with the $i$ th modal velocity being

$$
\dot{T}_{i}(0)=-\int_{0}^{a} \rho A W_{i}(x) F(x) v d x=v \sqrt{\rho A a} \frac{\Lambda_{i}}{\lambda_{i}}
$$

where $\Lambda_{i}=(-1)^{i} \sqrt{\sigma_{i}^{2}+1}+\sqrt{\sigma_{i}^{2}-1}$

Note that in Eq. (A8), the parameter $\Lambda_{i}$ is proportional to the $i$ th modal velocity, which is the coupling between the normal mode $W_{i}(x)$ representing the local vibration and the static motion of $F(x) v$. Values of $\Lambda_{i}$ are given in Table A.1. 
Table A.1 Solution of mode-dependent parameters for fixed-pinned boundary condition

\begin{tabular}{cccc}
\hline Mode number & $\lambda_{i}$ & $\sigma_{i}$ & $\Lambda_{i}$ \\
\hline 1 & 3.92660231 & 1.00077731 & -1.37532693 \\
2 & 7.06858275 & 1.00000145 & 1.41591746 \\
3 & 10.21017612 & 1.00000000 & -1.41413998 \\
4 & 13.35176878 & 1.00000000 & 1.41421674 \\
5 & 16.49336143 & 1.00000000 & -1.41421342 \\
$i>5$ & $(4 i+1) \pi / 4$ & 1.00000000 & $(-1)^{i} \sqrt{2}$ \\
\hline
\end{tabular}

Appendix B. Solutions of frequency equation of beam with fixed-fixed boundary conditions

The frequency equation of a beam with fixed-fixed boundary conditions is $\cos \left(\lambda_{i}^{\prime}\right) \cosh \left(\lambda_{i}^{\prime}\right)-1=0[31]$. Values of $\lambda_{i}^{\prime}$ are given in Table B.1.

Table B.1 Values of $\lambda_{i}^{\prime}$ for $i$ th vibration mode

\begin{tabular}{cc}
\hline Mode number & $\lambda_{i}^{\prime}$ \\
\hline 1 & 4.73004074 \\
2 & 7.85320462 \\
3 & 10.9956079 \\
4 & 14.1371655 \\
5 & 17.2787597 \\
$i>5$ & $(2 i+1) \pi / 2$ \\
\hline
\end{tabular}

\section{Data availability}

The authors confirm that the data supporting the findings of this study are available within the article.

\section{References}

[1] ASTM D5528-01, Standard test method for mode I interlaminar fracture toughness of unidirectional fiber-reinforced polymer matrix composites. 2014.

[2] D. E. Lambert and C. A. Ross, "Strain rate effects on dynamic fracture and strength," Int. J. Impact Eng., vol. 24, pp. 985-998, 2000.

[3] J. L. Tsai, C. Guo, and C. T. Sun, "Dynamic delamination fracture toughness in unidirectional polymeric composites," Compos. Sci. Technol., vol. 61, pp. 87-94, 2001.

[4] H. Zabala, L. Aretxabaleta, G. Castillo, and J. Aurrekoetxea, "Loading rate dependency 
on mode I interlaminar fracture toughness of unidirectional and woven carbon fibre epoxy composites," Compos. Struct., vol. 121, pp. 75-82, 2015.

[5] A. J. Smiley and R. B. Pipes, "Rate effects on mode I interlaminar fracture toughness in composite materials,” J. Compos. Mater., vol. 21, no. 7, pp. 670-687, 1987.

[6] B. R. K. Blackman et al., "The failure of fibre composites and adhesively bonded fibre composites under high rates of test: Part I Mode I loading - experimental studies," $J$. Mater. Sci., vol. 30, pp. 5885-5900, 1995.

[7] B. R. K. Blackman, A. J. Kinloch, Y. Wang, and J. G. Williams, "The failure of fibre composites and adhesively bonded fibre composites under high rates of test: Part II mode I loading - Dynamic effects,” J. Mater. Sci., vol. 31, pp. 4451-4466, 1996.

[8] H. Liu, H. Nie, C. Zhang, and Y. Li, "Loading rate dependency of Mode I interlaminar fracture toughness for unidirectional composite laminates," Compos. Sci. Technol., vol. 167, pp. 215-223, 2018.

[9] Y. Liu, F. P. van der Meer, and L. J. Sluys, "Cohesive zone and interfacial thick level set modeling of the dynamic double cantilever beam test of composite laminate," Theor. Appl. Fract. Mech., vol. 96, pp. 617-630, 2018.

[10] P. Davidson and A. M. Waas, "Non-smooth mode I fracture of fibre-reinforced composites: An experimental, numerical and analytical study," Philos. Trans. R. Soc. A Math. Phys. Eng. Sci., vol. 370, pp. 1942-1965, 2012.

[11] M. May, "Measuring the rate-dependent mode I fracture toughness of composites - A review," Compos. Part A Appl. Sci. Manuf., vol. 81, pp. 1-12, 2016.

[12] J. H. Song, H. Wang, and T. Belytschko, "A comparative study on finite element methods for dynamic fracture," Comput. Mech., vol. 42, pp. 239-250, 2008.

[13] T. Crump, G. Ferté, A. Jivkov, P. Mummery, and V. X. Tran, "Dynamic fracture analysis by explicit solid dynamics and implicit crack propagation," Int. J. Solids Struct., vol. 110-111, pp. 113-126, 2017.

[14] T. Chen, C. M. Harvey, S. Wang, and V. V. Silberschmidt, "Dynamic interfacial fracture of a thin-layered structure," Procedia Struct. Integr., vol. 13, pp. 613-618, 2018.

[15] T. Chen, C. M. Harvey, S. Wang, and V. V. Silberschmidt, "Dynamic interfacial fracture of a double cantilever beam," Eng. Fract. Mech., vol. 225, 2020.

[16] T. Chen, C. M. Harvey, S. Wang, and V. V. Silberschmidt, "Dynamic delamination on elastic interface," Compos. Struct., vol. 234, p. 111670, 2020.

[17] E. E. Gdoutos, Fracture Mechanics. Springer, 2005.

[18] L.B.Freund, Dynamic fracture mechanics. Cambridge University Press, 1990. 
[19] K.F.Graff, Wave Motion in Elastic Solids. Oxford University Press, 1991.

[20] S.Gopalakrishnan, Wave Propagation in Materials and Structures. CRC Press, 2017.

[21] N. L. Balazs, "On the solution of the wave equation with moving boundaries," J. Math. Anal. Appl., vol. 3, pp. 472-484, 1961.

[22] L. Gaffour, "Analytical method for solving the one-dimensional wave equation with moving boundary - abstract," J. Electromagn. Waves Appl., vol. 20, pp. 63-73, 1998.

[23] B. Pelloni and D. A. Pinotsis, "Moving boundary value problems for the wave equation," J. Comput. Appl. Math., vol. 234, pp. 1685-1691, 2010.

[24] I. C. Christov and C. I. Christov, "On mechanical waves and Doppler shifts from moving boundaries," Math. Methods Appl. Sci., vol. 40, no. 12, pp. 4481-4492, 2017.

[25] C. Hakoda and C. J. Lissenden, "Comparison of quasi-Rayleigh waves and Rayleigh waves, and clarifying the cut-off frequency of quasi-Rayleigh waves," Ultrasonics, vol. 92, pp. 50-56, 2019.

[26] V. Giurgiutiu, Structural health monitoring with piezoelectric wafer active sensors. Elsevier, 2007.

[27] H. Liu, X. Meng, H. Zhang, H. Nie, C. Zhang, and Y. Li, "The dynamic crack propagation behavior of mode I interlaminar crack in unidirectional carbon/epoxy composites," Eng. Fract. Mech., vol. 215, pp. 65-82, 2019.

[28] I. A. Viktorov, Rayleigh and Lamb Waves. 1967.

[29] D. R. Curran, L. Seaman, and D. A. Shockey, "Dynamic failure of solids," Phys. Rep., vol. 147, no. 5-6, pp. 253-388, 1987.

[30] D. A. Grant, "Beam vibrations with time-dependent boundary conditions," J. Sound Vib., vol. 89, no. 4, pp. 519-522, 1983.

[31] S. S. Rao, Vibration of Continuous Systems. John Wiley \& Sons, 2007. 